\title{
Article
}

\section{Post-Quantum Chebyshev-Type Integral Inequalities for Synchronous Functions}

\author{
Nuttapong Arunrat ${ }^{1}\left(\mathbb{D}\right.$, Keaitsuda Maneeruk Nakprasit $^{1}$ (D), Kamsing Nonlaopon ${ }^{1, *(D)}$, Praveen Agarwal ${ }^{2}$ (D) \\ and Sotiris K. Ntouyas ${ }^{3,4}$ (D) \\ 1 Department of Mathematics, Faculty of Science, Khon Kaen University, Khon Kaen 40002, Thailand; \\ nutaru@kkumail.com (N.A.); keaina@kku.ac.th (K.M.N.) \\ 2 Department of Mathematics, Anand International College of Engineering, Jaipur 302029, India; \\ goyal.praveen2011@gmail.com \\ 3 Department of Mathematics, University of Ioannina, 45110 Ioannina, Greece; sntouyas@uoi.gr \\ 4 Nonlinear Analysis and Applied Mathematics (NAAM)-Research Group, Department of Mathematics, \\ Faculty of Science, King Abdulaziz University, P.O. Box 80203, Jeddah 21589, Saudi Arabia \\ * Correspondence: nkamsi@kku.ac.th; Tel.: +668-6642-1582
}

Citation: Arunrat N.; Nakprasit K.M.; Nonlaopon, K.; Agarwal, P.; Ntouyas, S.K. Post-Quantum Chebyshev-Type Integral Inequalities for Synchronous Functions. Mathematics 2022, 10, 468. https:// doi.org/10.3390/math10030468 Academic Editor: Simeon Reich

Received: 16 December 2021 Accepted: 30 January 2022 Published: 31 January 2022

Publisher's Note: MDPI stays neutral with regard to jurisdictional claims in published maps and institutional affiliations.

Copyright: (C) 2022 by the authors. Licensee MDPI, Basel, Switzerland. This article is an open access article distributed under the terms and conditions of the Creative Commons Attribution (CC BY) license (https:/ / creativecommons.org/licenses/by/ $4.0 /)$.
Abstract: In this paper, we apply $(p, q)$-calculus to establish some new Chebyshev-type integral inequalities for synchronous functions. In particular, we generalize results of quantum Chebyshevtype integral inequalities by using $(p, q)$-integral. By taking $p=1$ and $q \rightarrow 1$, our results reduce to classical results on Chebyshev-type inequalities for synchronous functions. Furthermore, we consider their relevance with other related known results.

Keywords: Chebyshev-type inequalities; synchronous (asynchronous) functions; $(p, q)$-calculus; $(p, q)$-derivative; $(p, q)$-integral

MSC: 05A30; 34A08; 26D10; 26D15

\section{Introduction}

Let $f, g:[a, b] \rightarrow \mathbb{R}$ be integrable functions and $\phi, \varphi:[a, b] \rightarrow(0, \infty)$ be integrable functions. The Chebyshev functional is defined by

$$
\begin{aligned}
T(f, g, \phi, \varphi)= & \left(\int_{a}^{b} \varphi(x) d x\right)\left(\int_{a}^{b} \phi(x) f(x) g(x) d x\right) \\
& +\left(\int_{a}^{b} \phi(x) d x\right)\left(\int_{a}^{b} \varphi(x) f(x) g(x) d x\right) \\
& -\left(\int_{a}^{b} \varphi(x) f(x) d x\right)\left(\int_{a}^{b} \phi(x) g(x) d x\right) \\
& -\left(\int_{a}^{b} \phi(x) f(x) d x\right)\left(\int_{a}^{b} \varphi(x) g(x) d x\right)
\end{aligned}
$$

see [1] for more details.

We say that functions $f$ and $g$ are synchronous (asynchronous, respectively) on $[a, b]$ if $(f(x)-f(y))(g(x)-g(y)) \geq 0((f(x)-f(y))(g(x)-g(y)) \leq 0$, respectively) for each $x, y \in[a, b]$. It follows that $T(f, g, \phi, \varphi) \geq 0(T(f, g, \phi, \varphi) \leq 0$, respectively) if $f$ and $g$ are synchronous (asynchronous, respectively) on $[a, b]$ (see, e.g., [2,3]). The Chebyshev inequality can obtained from (1) by setting $\phi=\varphi$.

The Chebyshev functional (1) has attracted the attention of many researchers mainly due to its applications in numerical quadrature, probability, transform theory, and statistical problems. Moreover, the Chebyshev functional (1) has been applied to some integral inequalities by using known fractional integral operators, see [4-10] and the references therein. 
Quantum calculus, also known as q-calculus, was introduced by the mathematician Euler in the eighteenth century, studying calculus without limits, where classical mathematical formulas are obtained as $q \rightarrow 1$. Newton defined the number in $q$-infinite series. The definite $q$-integral, known as the $q$-Jackson integral, is defined by F. H. Jackson $[11,12]$ in 1910. In quantum calculus, it has many applications in various fields of mathematics and physics, specifically in hypergeometric functions, convexity function, orthogonal polynomials, mechanics, number theory, and relativity theory. In [13], M. Aslam et al. obtained quantum Ostrowski inequalities for the $q$-differentiable convex function. A. Aral et al. [14] studied applications of $q$-calculus in operator theory, and H. Gauchman [15] obtained integral inequalities in q-calculus. B. Ahmad et al. [16-19] studied boundary-value problems for nonlinear $q$-difference equations with any boundary conditions. In [20], J. D. BukweliKyemba and M. N. Hounkonnou studied quantum deformed algebra: coherent states and special functions. S. Bermudo et al. [21] obtained $q$-Hermite-Hadamard inequalities for general convex functions and F. Chen W. Yang [22] obtained some new Chebyshev-type quantum integral inequalities on finite intervals. Furthermore, the fundamental knowledge and theoretical concepts of quantum calculus are covered in the book written by V. Kac and P. Cheung [23].

In 2013, J. Tariboon and S. K. Ntouyas [24] introduced the quantum calculus on finite intervals. Next, they extended the Hölder, Hermite-Hadamard, trapezoid, Ostrowski, Cauchy-Bunyakovsky-Schwarz, Grüss, and Grüss-Čebyšev integral inequalities to quantum calculus on finite intervals in [25].

In 2016, M. Tunç and E. Göv [26,27] introduced the $(p, q)$-derivative and $(p, q)$-integral on finite intervals while proving some properties, and gave several inequalities of integral via $(p, q)$-calculus. In recent years, many quantum integral inequalities on a finite interval have been investigated more generally in $(p, q)$-calculus, which was first considered by R. Chakrabarti and R. Jagannathan [28]. It is worth noting that $q$-calculus cannot be directly retrieved by replacing $q$ with $q / p$ in $q$-calculus, but $q$-calculus may be recaptured by setting $p=1$ in $(p, q)$-calculus. In [29], J. Prabseang et al. obtained $(p, q)$-HermiteHadamard inequalities for double integral and $(p, q)$-differentiable convex functions. $\mathrm{H}$. Kalsoom et al. [30] obtained $(p, q)$-estimates of Hermite-Hadamard-type inequalities for coordinated convex and quasi-convex functions. In [31], M. N. Hounkonnou studied $(p, q)$-calculus: differentiation and integration and P. N. Sadjung [32] studied $(p, q)$-Taylor formula. Y. M. Chu et al. [33] obtained new post-quantum analogues of Ostrowski-type inequalities using new definitions of left-right $(p, q)$-derivatives and definite integrals. In [34], H. Kalsoom et al. obtained post-quantum Hermite-Hadamard type inequalities associated with coordinated higher-order generalized strongly pre-index and quasi-preindex mappings. M. Kunt et al. [35] obtained $(p, q)$-estimates of Hermite-Hadamard-type inequalities for midpoint type integral inequalities via convex and quasi convex function. In addition, more results of $(p, q)$-calculus appear in [36-39] and the references cited therein.

Motivated by the results mentioned above, by using the four parameters of deformation $\left(p_{1}, q_{1}\right)$ and $\left(p_{2}, q_{2}\right)$, we propose generalizing and extending some new Chebyshev inequalities in $q$-integral to $(p, q)$-integral. Furthermore, we obtain their relevance with other related known results. We hope that the ideas and techniques presented in this paper will inspire interested readers working in this field.

\section{Preliminaries}

In this section, we present basic concepts of $(p, q)$-calculus, which will be used in our work. Throughout this paper, we let $J=[a, b] \subseteq \mathbb{R}, K=[c, d] \subseteq \mathbb{R}, 0<q<p \leq 1,0<q_{i}<$ $p_{i} \leq 1(i=1,2)$ be constants, and we set ${ }_{a} b_{p}=p b+(1-p) a, b_{q}=q b+(1-q) a, c d_{p}=$ $p d+(1-p) c,{ }_{a} x_{p}=p x+(1-p) a$, and ${ }_{a} x_{q}=q x+(1-q) a$. 
Definition 1 ([26,27]). The $(p, q)$-derivative of a continuous function $f: J \rightarrow \mathbb{R}$ at $x$, denoted by ${ }_{a} D_{p, q} f(x)$, is defined by

$$
{ }_{a} D_{p, q} f(x)= \begin{cases}\frac{f\left({ }_{a} x_{p}\right)-f\left({ }_{a} x_{q}\right)}{(p-q)(x-a)}, & x \neq a ; \\ \lim _{x \rightarrow a} a D_{p, q} f(x), & x=a .\end{cases}
$$

If ${ }_{a} D_{p, q} f(x)$ exists for all $x \in J$, then $f$ is $(p, q)$-differentiable on $J$.

By setting $a=0$ in Definition 1 , we obtain ${ }_{0} D_{p, q} f=D_{p, q} f$, where $D_{p, q} f$ is defined by

$$
D_{p, q} f(x)= \begin{cases}\frac{f(p x)-f(q x)}{(p-q)(x)}, & x \neq 0 ; \\ \lim _{x \rightarrow 0} D_{p, q} f(x), & x=0 .\end{cases}
$$

In case $p=1,{ }_{a} D_{p, q} f={ }_{a} D_{q} f$ which is the $q$-derivative of the function $f$.

Example 1. For $x \in J$ and $n \in \mathbb{N}$, we have

$$
{ }_{a} D_{p, q}\left((x-a)^{n}\right)=\frac{p^{n}-q^{n}}{p-q} \cdot(x-a)^{n-1} .
$$

Definition $2([26,27])$. If $f: J \rightarrow \mathbb{R}$ is a continuous function and $x \in J$, then the $(p, q)$-integral of the function $f$ for $x$, denoted by $I_{p, q}^{a} f(x)$, is defined by

$$
I_{p, q}^{a} f(x):=\int_{a}^{x} f(t){ }_{a} d_{p, q} t=(p-q)(x-a) \sum_{n=0}^{\infty} \frac{q^{n}}{p^{n+1}} f\left(\frac{q^{n}}{p^{n+1}} x+\left(1-\frac{q^{n}}{p^{n+1}}\right) a\right) .
$$

Moreover, if $c \in(a, x)$, then the $(p, q)$-integral is defined by

$$
\int_{c}^{x} f(t)_{a} d_{p, q} t=\int_{a}^{x} f(t)_{a} d_{p, q} t-\int_{a}^{c} f(t)_{a} d_{p, q} t
$$

If $I_{p, q}^{a} f(x)$ exists for all $x \in J$, then $f$ is $(p, q)$-integrable on $J$.

Definition 2 reduces to the classical $q$-integral of the function $f$ when $a=0$ and $p=1$.

Example 2. Define a function $f: J \rightarrow \mathbb{R}$ by $f(x)=k x$, where $k \in \mathbb{R}$. Then

$$
\begin{aligned}
\int_{a}^{x} f(t){ }_{a} d_{p, q} t & =\int_{a}^{x} k t_{a} d_{p, q} t \\
& =(p-q)(x-a) \sum_{n=0}^{\infty} \frac{q^{n}}{p^{n+1}}\left[k\left(\frac{q^{n}}{p^{n+1}} x+\left(1-\frac{q^{n}}{p^{n+1}}\right) a\right)\right] \\
& =\frac{k(x-a)(x-a(1-p-q))}{p+q}
\end{aligned}
$$

Theorem $1([26,27])$. If $f: J \rightarrow \mathbb{R}$ is a continuous function and $c \in(a, x)$, then

(i) $\quad \int_{a}^{x}{ }_{a} D_{p, q} f(t){ }_{a} d_{p, q} t=f(x)-f(a)$;

(ii) $\quad \int_{c}^{x}{ }_{a} D_{p, q} f(t){ }_{a} d_{p, q} t=f(x)-f(c)$.

Theorem 2 ([26,27]). If $f, g: J \rightarrow \mathbb{R}$ are continuous functions, $x \in J$, and $\alpha \in \mathbb{R}$, then

(i) $\int_{a}^{x}[f(t)+g(t)]_{a} d_{p, q} t=\int_{a}^{x} f(t){ }_{a} d_{p, q} t+\int_{a}^{x} g(t)_{a} d_{p, q} t$;

(ii) $\int_{a}^{x} \alpha f(t)_{a} d_{p, q} t=\alpha \int_{a}^{x} f(t)_{a} d_{p, q} t$; 
(iii) $\int_{a}^{x} f(p t+(1-p) a)_{a} D_{p, q} g(t){ }_{a} d_{p, q} t=[(f g)(t)]_{a}^{x}-\int_{a}^{x} g(q t+(1-q) a)_{a} D_{p, q} f(t)_{a} d_{p, q} t$.

Lemma 1. If $f, g: J \rightarrow \mathbb{R}$ are continuous functions with $f(t) \leq g(t)$ for all $t \in J$, then, for $x \in[a, p b+(1-p) a]$, we have

$$
\int_{a}^{x} f(t){ }_{a} d_{p, q} t \leq \int_{a}^{x} g(t){ }_{a} d_{p, q} t .
$$

Proof. Let $x \in[a, p b+(1-p) a]$. Then $\frac{q^{n}}{p^{n+1}} x+\left(1-\frac{q^{n}}{p^{n+1}}\right) a \in[a, b]$. Because $f, g: J \rightarrow$ $\mathbb{R}$ are continuous functions with $f(t) \leq g(t)$ for all $t \in J$, we get

$$
f\left(\frac{q^{n}}{p^{n+1}} x+\left(1-\frac{q^{n}}{p^{n+1}}\right) a\right) \leq g\left(\frac{q^{n}}{p^{n+1}} x+\left(1-\frac{q^{n}}{p^{n+1}}\right) a\right) .
$$

We multiply (3) by $(p-q)(x-a) \cdot \frac{q^{n}}{p^{n+1}} \geq 0$ and then sum $n$ from zero to infinity to obtain

$$
\begin{aligned}
\int_{a}^{x} f(t){ }_{a} d_{p, q} t & =(p-q)(x-a) \sum_{n=0}^{\infty} \frac{q^{n}}{p^{n+1}} f\left(\frac{q^{n}}{p^{n+1}} x+\left(1-\frac{q^{n}}{p^{n+1}}\right) a\right) \\
& \leq(p-q)(x-a) \sum_{n=0}^{\infty} \frac{q^{n}}{p^{n+1}} g\left(\frac{q^{n}}{p^{n+1}} x+\left(1-\frac{q^{n}}{p^{n+1}}\right) a\right) \\
& =\int_{a}^{x} g(t){ }_{a} d_{p, q} t,
\end{aligned}
$$

which is inequality (2). This complete the proof.

The next lemma is due to N. Arunrat et al. [39].

Lemma 2 ([39]). If $f: J \rightarrow \mathbb{R}$ is a twice $(p, q)$-differentiable function with ${ }_{a} D_{p, q}^{2} f(p, q)$ integrable on $J$, then

$$
\begin{aligned}
\int_{a}^{a} b_{p} & (x-a)(b-x)_{a} D_{p, q}^{2} f(x)_{a} d_{p, q} x \\
& =\frac{q}{p^{2}}(b-a) f\left({ }_{a} b_{p q}\right)+\frac{1}{p}(b-a) f(a)-\left(\frac{p+q}{p^{3}}\right) \int_{a}^{a} b_{p} f\left({ }_{a} x_{q^{2}}\right){ }_{a} d_{p, q} x .
\end{aligned}
$$

\section{Main Results}

In this section, we establish some new Chebyshev-type integral inequalities via $(p, q)$ calculus. From now on, we assume that all of $(p, q)$-integral exists, and let

$$
I_{p, q}^{a}(u f)\left(a b_{p}\right):=\int_{a}^{a} b_{p} u(t) f(t){ }_{a} d_{p, q} t
$$

and

$$
I_{p, q}^{a}(u f g)\left(a b_{p}\right):=\int_{a}^{a b_{p}} u(t) f(t) g(t)_{a} d_{p, q} t
$$

Lemma 3. Let $f$ and $g$ be continuous and synchronous functions on $J$ and let $u, v: J \rightarrow[0, \infty)$ be continuous functions. Then

$$
\begin{aligned}
& I_{p, q}^{a} u\left({ }_{a} b_{p}\right) I_{p, q}^{a}(v f g)\left({ }_{a} b_{p}\right)+I_{p, q}^{a} v\left({ }_{a} b_{p}\right) I_{p, q}^{a}(u f g)\left({ }_{a} b_{p}\right) \\
& \quad \geq I_{p, q}^{a}(u f)\left({ }_{a} b_{p}\right) I_{p, q}^{a}(v g)\left({ }_{a} b_{p}\right)+I_{p, q}^{a}(v f)\left({ }_{a} b_{p}\right) I_{p, q}^{a}(u g)\left({ }_{a} b_{p}\right) .
\end{aligned}
$$


Proof. Since $f$ and $g$ are continuous and synchronous functions on $[a, b]$, we get for each $\alpha, \beta \in[a, b]$ that $(f(\alpha)-f(\beta))(g(\alpha)-g(\beta)) \geq 0$. Equivalently,

$$
f(\alpha) g(\alpha)+f(\beta) g(\beta) \geq f(\alpha) g(\beta)+f(\beta) g(\alpha) .
$$

Multiplying both sides of (5) by $v(\alpha)$ and then $(p, q)$-integrating the resulting relation with respect to $\alpha$ from $a$ to $a b_{p}$, we obtain

$$
I_{p, q}^{a}(v f g)\left({ }_{a} b_{p}\right)+f(\beta) g(\beta) I_{p, q}^{a} v\left({ }_{a} b_{p}\right) \geq g(\beta) I_{p, q}^{a}(v f)\left({ }_{a} b_{p}\right)+f(\beta) I_{p, q}^{a}(v g)\left(a_{a} b_{p}\right) .
$$

Multiplying both sides of (6) by $u(\beta)$ and then $(p, q)$-integrating the resulting relation with respect to $\beta$ from $a$ to $a b_{p}$, we obtain

$$
\begin{aligned}
I_{p, q}^{a} u\left({ }_{a} b_{p}\right) I_{p, q}^{a}(v f g)\left({ }_{a} b_{p}\right)+I_{p, q}^{a} v\left({ }_{a} b_{p}\right) I_{p, q}^{a}(u f g)\left({ }_{a} b_{p}\right) \\
\\
\geq I_{p, q}^{a}(u f)\left(a b_{p}\right) I_{p, q}^{a}(v g)\left({ }_{a} b_{p}\right)+I_{p, q}^{a}(v f)\left({ }_{a} b_{p}\right) I_{p, q}^{a}(u g)\left({ }_{a} b_{p}\right),
\end{aligned}
$$

which is inequality (4). The proof is complete.

Theorem 3. Let $f$ and $g$ be continuous and synchronous functions on $J$ and let $\phi, \varphi, \psi: J \rightarrow[0, \infty)$ be continuous functions. Then,

$$
\begin{aligned}
& 2 I_{p, q}^{a} \phi\left({ }_{a} b_{p}\right)\left[I_{p, q}^{a} \varphi\left({ }_{a} b_{p}\right) I_{p, q}^{a}(\psi f g)\left({ }_{a} b_{p}\right)+I_{p, q}^{a} \psi\left({ }_{a} b_{p}\right) I_{p, q}^{a}(\varphi f g)\left({ }_{a} b_{p}\right)\right] \\
&+2 I_{p, q}^{a} \varphi\left({ }_{a} b_{p}\right) I_{p, q}^{a} \psi\left({ }_{a} b_{p}\right) I_{p, q}^{a}(\phi f g)\left({ }_{a} b_{p}\right) \\
& \geq I_{p, q}^{a} \phi\left({ }_{a} b_{p}\right)\left[I_{p, q}^{a}(\varphi f)\left({ }_{a} b_{p}\right) I_{p, q}^{a}(\psi g)\left({ }_{a} b_{p}\right)\right. \\
&\left.+I_{p, q}^{a}(\psi f)\left({ }_{a} b_{p}\right) I_{p, q}^{a}(\varphi g)\left({ }_{a} b_{p}\right)\right] \\
&+I_{p, q}^{a} \varphi\left({ }_{a} b_{p}\right)\left[I_{p, q}^{a}(\phi f)\left({ }_{a} b_{p}\right) I_{p, q}^{a}(\psi g)\left({ }_{a} b_{p}\right)\right. \\
&\left.+I_{p, q}^{a}(\psi f)\left({ }_{a} b_{p}\right) I_{p, q}^{a}(\phi g)\left({ }_{a} b_{p}\right)\right] \\
&+I_{p, q}^{a} \psi\left({ }_{a} b_{p}\right)\left[I_{p, q}^{a}(\phi f)\left({ }_{a} b_{p}\right) I_{p, q}^{a}(\varphi g)\left({ }_{a} b_{p}\right)\right. \\
&\left.+I_{p, q}^{a}(\varphi f)\left({ }_{a} b_{p}\right) I_{p, q}^{a}(\phi g)\left({ }_{a} b_{p}\right)\right]
\end{aligned}
$$

Proof. Replacing $u$ and $v$ in (4) by $\varphi$ and $\psi$, respectively, we get

$$
\begin{aligned}
& I_{p, q}^{a} \varphi\left({ }_{a} b_{p}\right) I_{p, q}^{a}(\psi f g)\left({ }_{a} b_{p}\right)+I_{p, q}^{a} \psi\left({ }_{a} b_{p}\right) I_{p, q}^{a}(\varphi f g)\left({ }_{a} b_{p}\right) \\
& \quad \geq I_{p, q}^{a}(\varphi f)\left({ }_{a} b_{p}\right) I_{p, q}^{a}(\psi g)\left({ }_{a} b_{p}\right)+I_{p, q}^{a}(\psi f)\left({ }_{a} b_{p}\right) I_{p, q}^{a}(\varphi g)\left({ }_{a} b_{p}\right) .
\end{aligned}
$$

Multiplying both sides of (8) by $I_{p, q}^{a} \phi\left({ }_{a} b_{p}\right)$, we obtain

$$
\begin{gathered}
I_{p, q}^{a} \phi\left({ }_{a} b_{p}\right)\left[I_{p, q}^{a} \varphi\left({ }_{a} b_{p}\right) I_{p, q}^{a}(\psi f g)\left({ }_{a} b_{p}\right)+I_{p, q}^{a} \psi\left({ }_{a} b_{p}\right) I_{p, q}^{a}(\varphi f g)\left({ }_{a} b_{p}\right)\right] \\
\geq I_{p, q}^{a} \phi\left({ }_{a} b_{p}\right)\left[I_{p, q}^{a}(\varphi f)\left({ }_{a} b_{p}\right) I_{p, q}^{a}(\psi g)\left({ }_{a} b_{p}\right)\right. \\
\left.\quad+I_{p, q}^{a}(\psi f)\left({ }_{a} b_{p}\right) I_{p, q}^{a}(\varphi g)\left({ }_{a} b_{p}\right)\right] .
\end{gathered}
$$

By substituting $u=\phi$ and $v=\psi$ in (4), we have

$$
\begin{aligned}
& I_{p, q}^{a} \phi\left({ }_{a} b_{p}\right) I_{p, q}^{a}(\psi f g)\left({ }_{a} b_{p}\right)+I_{p, q}^{a} \psi\left(a b_{p}\right) I_{p, q}^{a}(\phi f g)\left({ }_{a} b_{p}\right) \\
& \quad \geq I_{p, q}^{a}(\phi f)\left({ }_{a} b_{p}\right) I_{p, q}^{a}(\psi g)\left({ }_{a} b_{p}\right)+I_{p, q}^{a}(\psi f)\left({ }_{a} b_{p}\right) I_{p, q}^{a}(\phi g)\left({ }_{a} b_{p}\right) .
\end{aligned}
$$


Multiplying both sides of (10) by $I_{p, q}^{a} \varphi\left({ }_{a} b_{p}\right)$, we obtain

$$
\begin{gathered}
I_{p, q}^{a} \varphi\left({ }_{a} b_{p}\right)\left[I_{p, q}^{a} \phi\left({ }_{a} b_{p}\right) I_{p, q}^{a}(\psi f g)\left({ }_{a} b_{p}\right)+I_{p, q}^{a} \psi\left({ }_{a} b_{p}\right) I_{p, q}^{a}(\phi f g)\left({ }_{a} b_{p}\right)\right] \\
\geq I_{p, q}^{a} \varphi\left({ }_{a} b_{p}\right)\left[I_{p, q}^{a}(\phi f)\left({ }_{a} b_{p}\right) I_{p, q}^{a}(\psi g)\left({ }_{a} b_{p}\right)\right. \\
\left.\quad+I_{p, q}^{a}(\psi f)\left({ }_{a} b_{p}\right) I_{p, q}^{a}(\phi g)\left({ }_{a} b_{p}\right)\right] .
\end{gathered}
$$

Putting $u=\phi$ and $v=\varphi$ in (4) and multiplying both sides by $I_{p, q}^{a} \psi\left(a_{a} b_{p}\right)$, we get

$$
\begin{aligned}
I_{p, q}^{a} \psi\left({ }_{a} b_{p}\right)\left[I_{p, q}^{a} \phi\left({ }_{a} b_{p}\right) I_{p, q}^{a}(\varphi f g)\left({ }_{a} b_{p}\right)+I_{p, q}^{a} \varphi\left({ }_{a} b_{p}\right) I_{p, q}^{a}(\phi f g)\left({ }_{a} b_{p}\right)\right] \\
\geq I_{p, q}^{a} \psi\left({ }_{a} b_{p}\right)\left[I_{p, q}^{a}(\phi f)\left({ }_{a} b_{p}\right) I_{p, q}^{a}(\varphi g)\left({ }_{a} b_{p}\right)\right. \\
\left.\quad+I_{p, q}^{a}(\varphi f)\left({ }_{a} b_{p}\right) I_{p, q}^{a}(\phi g)\left({ }_{a} b_{p}\right)\right] .
\end{aligned}
$$

By combining (9), (11), and (12), we obtain inequality (7), which accomplishes the proof.

\section{Remark 1.}

(i) Lemma 3 and Theorem 3 are reversed in the following cases.

(a) The functions $f$ and $g$ are asynchronous on $J$.

(b) The functions $\phi, \varphi$, and $\psi$ are non-positive on $J$.

(c) Two of the functions $\phi, \varphi$, and $\psi$ are non-negative and the third one is non-positive on $J$.

(ii) If we take $p=1$ in Theorem 3, then it reduces to Theorem 3.2 in [22].

Lemma 4. Let $f$ and $g$ be continuous and synchronous functions on $J \cup K$ and let $u, v: J \cup K \rightarrow$ $[0, \infty)$ be continuous functions. Then,

$$
\begin{gathered}
I_{p_{1}, q_{1}}^{a} u\left({ }_{a} b_{p_{1}}\right) I_{p_{2}, q_{2}}^{c}(v f g)\left({ }_{c} d_{p_{2}}\right)+I_{p_{1}, q_{1}}^{a}(u f g)\left({ }_{a} b_{p_{1}}\right) I_{p_{2}, q_{2}}^{c} v\left({ }_{c} d_{p_{2}}\right) \\
\geq I_{p_{1}, q_{1}}^{a}(u f)\left({ }_{a} b_{p_{1}}\right) I_{p_{2}, q_{2}}^{c}(v g)\left({ }_{c} d_{p_{2}}\right) \\
\quad+I_{p_{1}, q_{1}}^{a}(u g)\left({ }_{a} b_{p_{1}}\right) I_{p_{2}, q_{2}}^{c}(v f)\left({ }_{c} d_{p_{2}}\right) .
\end{gathered}
$$

Proof. Since $f$ and $g$ are continuous and synchronous functions on $J \cup K$, we get for all $\alpha, \beta \in J \cup K$ that $(f(\alpha)-f(\beta))(g(\alpha)-g(\beta)) \geq 0$. Equivalently,

$$
f(\alpha) g(\alpha)+f(\beta) g(\beta) \geq f(\alpha) g(\beta)+f(\beta) g(\alpha) .
$$

Multiplying both sides of (14) by $v(\beta)$ and then $\left(p_{2}, q_{2}\right)$-integrating the resulting inequality with respect to $\beta$ from $c$ to ${ }_{c} d_{p_{2}}$, we obtain

$$
\begin{aligned}
f(\alpha) g(\alpha) I_{p_{2}, q_{2}}^{c} v\left({ }_{c} d_{p_{2}}\right)+I_{p_{2}, q_{2}}^{c}(v f g)\left({ }_{c} d_{p_{2}}\right) \\
\quad \geq f(\alpha) I_{p_{2}, q_{2}}^{c}(v g)\left({ }_{c} d_{p_{2}}\right)+g(\alpha) I_{p_{2}, q_{2}}^{c}(v f)\left({ }_{c} d_{p_{2}}\right)
\end{aligned}
$$

Multiplying both sides of (15) by $u(\alpha)$ and then $\left(p_{1}, q_{1}\right)$-integrating the resulting inequality with respect to $\alpha$ from $a$ to ${ }_{a} b_{p_{1}}$, we obtain

$$
\begin{aligned}
& I_{p_{1}, q_{1}}^{a}(u f g)\left({ }_{a} b_{p_{1}}\right) I_{p_{2}, q_{2}}^{c} v\left({ }_{c} d_{p_{2}}\right)+I_{p_{1}, q_{1}}^{a} u\left({ }_{a} b_{p_{1}}\right) I_{p_{2}, q_{2}}^{c}(v f g)\left({ }_{c} d_{p_{2}}\right) \\
& \quad \geq I_{p_{1}, q_{1}}^{a}(u f)\left({ }_{a} b_{p_{1}}\right) I_{p_{2}, q_{2}}^{c}(v g)\left({ }_{c} d_{p_{2}}\right)+I_{p_{1}, q_{1}}^{a}(u g)\left({ }_{a} b_{p_{1}}\right) I_{p_{2}, q_{2}}^{c}(v f)\left({ }_{c} d_{p_{2}}\right),
\end{aligned}
$$

which is inequality (13). The proof is complete.

Theorem 4. Let $f$ and $g$ be continuous and synchronous functions on $J \cup K$ and let $\phi, \varphi, \psi$ : $J \cup K \rightarrow[0, \infty)$ be continuous functions. Then, 


$$
\begin{aligned}
& I_{p_{1}, q_{1}}^{a} \phi\left({ }_{a} b_{p_{1}}\right)\left[I_{p_{1}, q_{1}}^{a} \psi\left({ }_{a} b_{p_{1}}\right) I_{p_{2}, q_{2}}^{c}(\varphi f g)\left({ }_{c} d_{p_{2}}\right)+2 I_{p_{1}, q_{1}}^{a} \varphi\left({ }_{a} b_{p_{1}}\right) I_{p_{2}, q_{2}}^{c}(\psi f g)\left({ }_{c} d_{p_{2}}\right)\right. \\
&\left.+I_{p_{1}, q_{1}}^{a}(\varphi f g)\left({ }_{a} b_{p_{1}}\right) I_{p_{2}, q_{2}}^{c} \psi\left({ }_{c} d_{p_{2}}\right)\right] \\
&+I_{p_{1}, q_{1}}^{a}(\phi f g)\left({ }_{a} b_{p_{1}}\right)\left[I_{p_{1}, q_{1}}^{a} \varphi\left({ }_{a} b_{p_{1}}\right) I_{p_{2}, q_{2}}^{c} \psi\left({ }_{c} d_{p_{2}}\right)\right. \\
&\left.+I_{p_{1}, q_{1}}^{a} \psi\left({ }_{a} b_{p_{1}}\right) I_{p_{2}, q_{2}}^{c} \varphi\left({ }_{c} d_{p_{2}}\right)\right] \\
& \geq I_{p_{1}, q_{1}}^{a} \phi\left({ }_{a} b b_{p_{1}}\right)\left[I_{p_{1}, q_{1}}^{a}(\varphi f)\left({ }_{a} b_{p_{1}}\right) I_{p_{2}, q_{2}}^{c}(\psi g)\left({ }_{c} d_{p_{2}}\right)\right. \\
&\left.+I_{p_{1}, q_{1}}^{a}(\varphi g)\left({ }_{a} b_{p_{1}}\right) I_{p_{2}, q_{2}}^{c}(\psi f)\left({ }_{c} d_{p_{2}}\right)\right] \\
&+I_{p_{1}, q_{1}}^{a} \varphi\left({ }_{a} b_{p_{1}}\right)\left[I_{p_{1}, q_{1}}^{a}(\phi f)\left({ }_{a} b_{p_{1}}\right) I_{p_{2}, q_{2}}^{c}(\psi g)\left({ }_{c} d_{p_{2}}\right)\right. \\
&\left.+I_{p_{1}, q_{1}}^{a}(\phi g)\left({ }_{a} b_{p_{1}}\right) I_{p_{2}, q_{2}}^{c}(\psi f)\left({ }_{c} d_{p_{2}}\right)\right] \\
&+I_{p_{1}, q_{1}}^{a} \psi\left({ }_{a} b_{p_{1}}\right)\left[I_{p_{1}, q_{1}}^{a}(\phi f)\left({ }_{a} b_{p_{1}}\right) I_{p_{2}, q_{2}}^{c}(\varphi g)\left({ }_{c} d_{p_{2}}\right)\right. \\
&\left.+I_{p_{1}, q_{1}}^{a}(\phi g)\left({ }_{a} b_{p_{1}}\right) I_{p_{2}, q_{2}}^{c}(\varphi f)\left({ }_{c} d_{p_{2}}\right)\right] .
\end{aligned}
$$

Proof. Putting $u=\varphi$ and $v=\psi$ in (13), we get

$$
\begin{aligned}
& I_{p_{1}, q_{1}}^{a} \varphi\left({ }_{a} b_{p_{1}}\right) I_{p_{2}, q_{2}}^{c}(\psi f g)\left({ }_{c} d_{p_{2}}\right)+I_{p_{1}, q_{1}}^{a}(\varphi f g)\left({ }_{a} b_{p_{1}}\right) I_{p_{2}, q_{2}}^{c} \psi\left({ }_{c} d_{p_{2}}\right) \\
& \geq I_{p_{1}, q_{1}}^{a}(\varphi f)\left({ }_{a} b_{p_{1}}\right) I_{p_{2}, q_{2}}^{c}(\psi g)\left({ }_{c} d_{p_{2}}\right) \\
& \quad+I_{p_{1}, q_{1}}^{a}(\varphi g)\left({ }_{a} b_{p_{1}}\right) I_{p_{2}, q_{2}}^{c}(\psi f)\left({ }_{c} d_{p_{2}}\right) .
\end{aligned}
$$

Multiplying both sides of (17) by $I_{p_{1}, q_{1}}^{a} \phi\left(a_{a} b_{p_{1}}\right)$, we obtain

$$
\begin{aligned}
I_{p_{1}, q_{1}}^{a} \phi\left({ }_{a} b_{p_{1}}\right)\left[I_{p_{1}, q_{1}}^{a} \varphi\left({ }_{a} b_{p_{1}}\right) I_{p_{2}, q_{2}}^{c}(\psi f g)\left({ }_{c} d_{p_{2}}\right)+I_{p_{1}, q_{1}}^{a}(\varphi f g)\left({ }_{a} b_{p_{1}}\right) I_{p_{2}, q_{2}}^{c} \psi\left({ }_{c} d_{p_{2}}\right)\right] \\
\geq \\
\quad I_{p_{1}, q_{1}}^{a} \phi\left({ }_{a} b_{p_{1}}\right)\left[I_{p_{1}, q_{1}}^{a}(\varphi f)\left({ }_{a} b_{p_{1}}\right) I_{p_{2}, q_{2}}^{c}(\psi g)\left({ }_{c} d_{p_{2}}\right)\right. \\
\left.\quad+I_{p_{1}, q_{1}}^{a}(\varphi g)\left({ }_{a} b_{p_{1}}\right) I_{p_{2}, q_{2}}^{c}(\psi f)\left({ }_{c} d_{p_{2}}\right)\right] .
\end{aligned}
$$

By substituting $u=\phi$ and $v=\psi$ in (13), we have

$$
\begin{aligned}
& I_{p_{1}, q_{1}}^{a} \phi\left({ }_{a} b_{p_{1}}\right) I_{p_{2}, q_{2}}^{c}(\psi f g)\left({ }_{c} d_{p_{2}}\right)+I_{p_{1}, q_{1}}^{a}(\phi f g)\left({ }_{a} b_{p_{1}}\right) I_{p_{2}, q_{2}}^{c} \psi\left({ }_{c} d_{p_{2}}\right) \\
& \geq I_{p_{1}, q_{1}}^{a}(\phi f)\left({ }_{a} b_{p_{1}}\right) I_{p_{2}, q_{2}}^{c}(\psi g)\left({ }_{c} d_{p_{2}}\right) \\
& \quad+I_{p_{1}, q_{1}}^{a}(\phi g)\left({ }_{a} b_{p_{1}}\right) I_{p_{2}, q_{2}}^{c}(\psi f)\left({ }_{c} d_{p_{2}}\right) .
\end{aligned}
$$

Multiplying both sides of (19) by $I_{p_{1}, q_{1}}^{a} \varphi\left({ }_{a} b_{p_{1}}\right)$, we obtain

$$
\begin{aligned}
I_{p_{1}, q_{1}}^{a} \varphi\left({ }_{a} b_{p_{1}}\right)\left[I_{p_{1}, q_{1}}^{a} \phi\left({ }_{a} b_{p_{1}}\right) I_{p_{2}, q_{2}}^{c}(\psi f g)\left({ }_{c} d_{p_{2}}\right)+I_{p_{1}, q_{1}}^{a}(\phi f g)\left({ }_{a} b_{p_{1}}\right) I_{p_{2}, q_{2}}^{c} \psi\left({ }_{c} d_{p_{2}}\right)\right] \\
\geq I_{p_{1}, q_{1}}^{a} \varphi\left({ }_{a} b_{p_{1}}\right)\left[I_{p_{1}, q_{1}}^{a}(\phi f)\left({ }_{a} b_{p_{1}}\right) I_{p_{2}, q_{2}}^{c}(\psi g)\left({ }_{c} d_{p_{2}}\right)\right. \\
\left.\quad+I_{p_{1}, q_{1}}^{a}(\phi g)\left({ }_{a} b_{p_{1}}\right) I_{p_{2}, q_{2}}^{c}(\psi f)\left({ }_{c} d_{p_{2}}\right)\right] .
\end{aligned}
$$
we get

Putting $u=\phi$ and $v=\varphi$ in (13) and then multiplying both sides by $I_{p_{1}, q_{1}}^{a} \psi\left({ }_{a} b_{p_{1}}\right)$, 


$$
\begin{gathered}
I_{p_{1}, q_{1}}^{a} \psi\left({ }_{a} b_{p_{1}}\right)\left[I_{p_{1}, q_{1}}^{a} \phi\left({ }_{a} b_{p_{1}}\right) I_{p_{2}, q_{2}}^{c}(\varphi f g)\left({ }_{c} d_{p_{2}}\right)+I_{p_{1}, q_{1}}^{a}(\phi f g)\left({ }_{a} b_{p_{1}}\right) I_{p_{2}, q_{2}}^{c} \varphi\left({ }_{c} d_{p_{2}}\right)\right] \\
\geq I_{p_{1}, q_{1}}^{a} \psi\left({ }_{a} b_{p_{1}}\right)\left[I_{p_{1}, q_{1}}^{a}(\phi f)\left({ }_{a} b_{p_{1}}\right) I_{p_{2}, q_{2}}^{c}(\varphi g)\left({ }_{c} d_{p_{2}}\right)\right. \\
\left.\quad+I_{p_{1}, q_{1}}^{a}(\phi g)\left({ }_{a} b_{p_{1}}\right) I_{p_{2}, q_{2}}^{c}(\varphi f)\left({ }_{c} d_{p_{2}}\right)\right] .
\end{gathered}
$$
proof.

Inequality (16) can obtained by combining (18), (20), and (21). We accomplish the

\section{Remark 2.}

(i) Theorem 4 is reversed in the following cases.

(a) The functions $f$ and $g$ are asynchronous on $J \cup K$.

(b) The functions $\phi, \varphi$, and $\psi$ are non-positive on $J \cup K$.

(c) Two of the functions $\phi, \varphi$, and $\psi$ are non-negative, and the third one is non-positive on $J \cup K$.

(ii) Theorem 3.4 in [22] is a special case of Theorem 4, when $p_{1}=p_{2}=1$.

Theorem 5. Let $f, g, h$ be continuous and synchronous functions on $J \cup K$ and let $u: J \cup K \rightarrow$ $[0, \infty)$ be a continuous function. Then,

$$
\begin{aligned}
& I_{p_{1}, q_{1}}^{a} u\left({ }_{a} b_{p_{1}}\right) I_{p_{2}, q_{2}}^{c}(u f g h)\left({ }_{c} d_{p_{2}}\right)+I_{p_{1}, q_{1}}^{a}(u h)\left({ }_{a} b_{p_{1}}\right) I_{p_{2}, q_{2}}^{c}(u f g)\left({ }_{c} d_{p_{2}}\right) \\
& \quad+I_{p_{1}, q_{1}}^{a}(u f g)\left(a_{a} b_{p_{1}}\right) I_{p_{2}, q_{2}}^{c}(u h)\left({ }_{c} d_{p_{2}}\right)+I_{p_{1}, q_{1}}^{a}(u f g h)\left({ }_{a} b_{p_{1}}\right) I_{p_{2}, q_{2}}^{c} u\left({ }_{c} d_{p_{2}}\right) \\
& \geq \\
& \quad I_{p_{1}, q_{1}}^{a}(u f)\left({ }_{a} b_{p_{1}}\right) I_{p_{2}, q_{2}}^{c}(u g h)\left({ }_{c} d_{p_{2}}\right)+I_{p_{1}, q_{1}}^{a}(u f h)\left({ }_{a} b_{p_{1}}\right) I_{p_{2}, q_{2}}^{c}(u g)\left({ }_{c} d_{p_{2}}\right) \\
& \quad+I_{p_{1}, q_{1}}^{a}(u g)\left({ }_{a} b_{p_{1}}\right) I_{p_{2}, q_{2}}^{c}(u f h)\left({ }_{c} d_{p_{2}}\right) \\
& \quad+I_{p_{1}, q_{1}}^{a}(u g h)\left({ }_{a} b_{p_{1}}\right) I_{p_{2}, q_{2}}^{c}(u f)\left({ }_{c} d_{p_{2}}\right) .
\end{aligned}
$$

Proof. Let $f, g, h$ be continuous and synchronous functions on $J \cup K$. Then for each $\alpha, \beta \in$ $J \cup K,(f(\alpha)-f(\beta))(g(\alpha)-g(\beta))(h(\alpha)+h(\beta)) \geq 0$. Equivalently,

$$
\begin{aligned}
& f(\alpha) g(\alpha) h(\alpha)+f(\beta) g(\beta) h(\beta)+f(\alpha) g(\alpha) h(\beta)+f(\beta) g(\beta) h(\alpha) \\
& \quad \geq f(\alpha) g(\beta) h(\alpha)+f(\alpha) g(\beta) h(\beta)+f(\beta) g(\alpha) h(\alpha)+f(\beta) g(\alpha) h(\beta) .
\end{aligned}
$$

Multiplying both sides of (23) by $u(\alpha)$ and $\left(p_{2}, q_{2}\right)$-integrating the resulting inequality with respect to $\alpha$ from $c$ to ${ }_{c} d_{p_{2}}$, we obtain

$$
\begin{aligned}
I_{p_{2}, q_{2}}^{c}(u f g h)\left({ }_{c} d_{p_{2}}\right)+f(\beta) g(\beta) h(\beta) I_{p_{2}, q_{2}}^{c} u\left({ }_{c} d_{p_{2}}\right) \\
\quad+h(\beta) I_{p_{2}, q_{2}}^{c}(u f g)\left({ }_{c} d_{p_{2}}\right)+f(\beta) g(\beta) I_{p_{2}, q_{2}}^{c}(u h)\left({ }_{c} d_{p_{2}}\right) \\
\geq g(\beta) I_{p_{2}, q_{2}}^{c}(u f h)\left({ }_{c} d_{p_{2}}\right)+g(\beta) h(\beta) I_{p_{2}, q_{2}}^{c}(u f)\left({ }_{c} d_{p_{2}}\right) \\
\quad+f(\beta) I_{p_{2}, q_{2}}^{c}(u g h)\left({ }_{c} d_{p_{2}}\right)+f(\beta) h(\beta) I_{p_{2}, q_{2}}^{c}(u g)\left({ }_{c} d_{p_{2}}\right) .
\end{aligned}
$$

Multiplying both sides of (24) by $u(\beta)$ and $\left(p_{1}, q_{1}\right)$-integrating the resulting inequality, with respect to $\beta$ from $a$ to $a b_{p_{1}}$, we get

$$
\begin{aligned}
& I_{p_{1}, q_{1}}^{a} u\left({ }_{a} b_{p_{1}}\right) I_{p_{2}, q_{2}}^{c}(u f g h)\left({ }_{c} d_{p_{2}}\right)+I_{p_{1}, q_{1}}^{a}(u f g h)\left({ }_{a} b_{p_{1}}\right) I_{p_{2}, q_{2}}^{c} u\left({ }_{c} d_{p_{2}}\right) \\
& \quad+I_{p_{1}, q_{1}}^{a}(u h)\left({ }_{a} b_{p_{1}}\right) I_{p_{2}, q_{2}}^{c}(u f g)\left({ }_{c} d_{p_{2}}\right)+I_{p_{1}, q_{1}}^{a}(u f g)\left({ }_{a} b_{p_{1}}\right) I_{p_{2}, q_{2}}^{c}(u h)\left({ }_{c} d_{p_{2}}\right) \\
& \geq I_{p_{1}, q_{1}}^{a}(u g)\left({ }_{a} b_{p_{1}}\right) I_{p_{2}, q_{2}}^{c}(u f h)\left({ }_{c} d_{p_{2}}\right)+I_{p_{1}, q_{1}}^{a}(u g h)\left({ }_{a} b_{p_{1}}\right) I_{p_{2}, q_{2}}^{c}(u f)\left({ }_{c} d_{p_{2}}\right) \\
& \quad+I_{p_{1}, q_{1}}^{a}(u f)\left({ }_{a} b_{p_{1}}\right) I_{p_{2}, q_{2}}^{c}(u g h)\left({ }_{c} d_{p_{2}}\right)+I_{p_{1}, q_{1}}^{a}(u f h)\left({ }_{a} b_{p_{1}}\right) I_{p_{2}, q_{2}}^{c}(u g)\left({ }_{c} d_{p_{2}}\right),
\end{aligned}
$$

which is inequality (22). The proof is complete. 
Theorem 6. Let $f, g, h$ be continuous and synchronous functions on $J \cup K$ and let $u, v: J \cup K \rightarrow$ $[0, \infty)$ be continuous functions. Then,

$$
\begin{aligned}
& I_{p_{1}, q_{1}}^{a} u\left({ }_{a} b_{p_{1}}\right) I_{p_{2}, q_{2}}^{c}(v f g h)\left({ }_{c} d_{p_{2}}\right)+I_{p_{1}, q_{1}}^{a}(u h)\left({ }_{a} b_{p_{1}}\right) I_{p_{2}, q_{2}}^{c}(v f g)\left({ }_{c} d_{p_{2}}\right) \\
& \quad+I_{p_{1}, q_{1}}^{a}(u f g)\left({ }_{a} b_{p_{1}}\right) I_{p_{2}, q_{2}}^{c}(v h)\left({ }_{c} d_{p_{2}}\right)+I_{p_{1}, q_{1}}^{a}(u f g h)\left({ }_{a} b_{p_{1}}\right) I_{p_{2}, q_{2}}^{c} v\left({ }_{c} d_{p_{2}}\right) \\
& \geq I_{p_{1}, q_{1}}^{a}(u f)\left({ }_{a} b_{p_{1}}\right) I_{p_{2}, q_{2}}^{c}(v g h)\left({ }_{c} d_{p_{2}}\right)+I_{p_{1}, q_{1}}^{a}(u f h)\left({ }_{a} b_{p_{1}}\right) I_{p_{2}, q_{2}}^{c}(v g)\left({ }_{c} d_{p_{2}}\right) \\
& \quad+I_{p_{1}, q_{1}}^{a}(u g)\left({ }_{a} b_{p_{1}}\right) I_{p_{2}, q_{2}}^{c}(v f h)\left({ }_{c} d_{p_{2}}\right)+I_{p_{1}, q_{1}}^{a}(u g h)\left({ }_{a} b_{p_{1}}\right) I_{p_{2}, q_{2}}^{c}(v f)\left({ }_{c} d_{p_{2}}\right) .
\end{aligned}
$$

Proof. Let $f, g, h$ be continuous and synchronous functions on $J \cup K$. Then for each $\alpha, \beta \in$ $J \cup K,(f(\alpha)-f(\beta))(g(\alpha)-g(\beta))(h(\alpha)+h(\beta)) \geq 0$. This implies that

$$
\begin{aligned}
& f(\alpha) g(\alpha) h(\alpha)+f(\beta) g(\beta) h(\beta)+f(\alpha) g(\alpha) h(\beta)+f(\beta) g(\beta) h(\alpha) \\
& \quad \geq f(\alpha) g(\beta) h(\alpha)+f(\alpha) g(\beta) h(\beta)+f(\beta) g(\alpha) h(\alpha)+f(\beta) g(\alpha) h(\beta) .
\end{aligned}
$$

Multiplying both sides of (26) by $v(\alpha)$ and $\left(p_{2}, q_{2}\right)$-integrating the resulting inequality with respect to $\alpha$ from $c$ to $c d_{p_{2}}$, we obtain

$$
\begin{aligned}
& I_{p_{2}, q_{2}}^{c}(v f g h)\left({ }_{c} d_{p_{2}}\right)+f(\beta) g(\beta) h(\beta) I_{p_{2}, q_{2}}^{c} v\left({ }_{c} d_{p_{2}}\right) \\
& \quad+h(\beta) I_{p_{2}, q_{2}}^{c}(v f g)\left({ }_{c} d_{p_{2}}\right)+f(\beta) g(\beta) I_{p_{2}, q_{2}}^{c}(v h)\left({ }_{c} d_{p_{2}}\right) \\
& \geq g(\beta) I_{p_{2}, q_{2}}^{c}(v f h)\left({ }_{c} d_{p_{2}}\right)+g(\beta) h(\beta) I_{p_{2}, q_{2}}^{c}(v f)\left({ }_{c} d_{p_{2}}\right) \\
&+f(\beta) I_{p_{2}, q_{2}}^{c}(v g h)\left({ }_{c} d_{p_{2}}\right)+f(\beta) h(\beta) I_{p_{2}, q_{2}}^{c}(v g)\left({ }_{c} d_{p_{2}}\right) .
\end{aligned}
$$

Multiplying both sides of (27) by $u(\beta)$ and $\left(p_{1}, q_{1}\right)$-integrating the resulting inequality with respect to $\beta$ from $a$ to $a b_{p_{1}}$, we get

$$
\begin{aligned}
& I_{p_{1}, q_{1}}^{a} u\left({ }_{a} b_{p_{1}}\right) I_{p_{2}, q_{2}}^{c}(v f g h)\left({ }_{c} d_{p_{2}}\right)+I_{p_{1}, q_{1}}^{a}(u f g h)\left({ }_{a} b_{p_{1}}\right) I_{p_{2}, q_{2}}^{c} v\left({ }_{c} d_{p_{2}}\right) \\
& \quad+I_{p_{1}, q_{1}}^{a}(u h)\left({ }_{a} b_{p_{1}}\right) I_{p_{2}, q_{2}}^{c}(v f g)\left({ }_{c} d_{p_{2}}\right)+I_{p_{1}, q_{1}}^{a}(u f g)\left({ }_{a} b_{p_{1}}\right) I_{p_{2}, q_{2}}^{c}(v h)\left({ }_{c} d_{p_{2}}\right) \\
& \geq I_{p_{1}, q_{1}}^{a}(u g)\left({ }_{a} b_{p_{1}}\right) I_{p_{2}, q_{2}}^{c}(v f h)\left({ }_{c} d_{p_{2}}\right)+I_{p_{1}, q_{1}}^{a}(u g h)\left({ }_{a} b_{p_{1}}\right) I_{p_{2}, q_{2}}^{c}(v f)\left({ }_{c} d_{p_{2}}\right) \\
& \quad+I_{p_{1}, q_{1}}^{a}(u f)\left({ }_{a} b_{p_{1}}\right) I_{p_{2}, q_{2}}^{c}(v g h)\left({ }_{c} d_{p_{2}}\right)+I_{p_{1}, q_{1}}^{a}(u f h)\left({ }_{a} b_{p_{1}}\right) I_{p_{2}, q_{2}}^{c}(v g)\left({ }_{c} d_{p_{2}}\right),
\end{aligned}
$$

which is inequality (25). The proof is complete.

\section{Remark 3.}

(i) It may be noted that inequalities in Theorem 5 and 6 are reversed when functions $f, g$, and $h$ are asynchronous.

(ii) Theorem 5 can obtained from Theorem 6 by letting $u=v$.

(iii) If we take $p_{1}=1$ and $p_{2}=1$ in Theorem 5 and 6 , then they reduce to ([22], Theorems 3.6 and 3.7$)$, respectively.

In the next theorem, we give some inequalities of Fejér-type inequalities by applying $(p, q)$-calculus to the weighted Chebyshev-type inequality.

Theorem 7. Let $f: J \rightarrow \mathbb{R}$ be a twice $(p, q)$-differentiable with ${ }_{a} D_{p, q}^{2} f$-integrable on J. Assume that ${ }_{a} D_{p, q}^{2} f$ is non-constant and $m \leq{ }_{a} D_{p, q}^{2} f \leq M$. Then

$$
\begin{aligned}
\frac{M p^{2} q^{2}(b-a)^{3}}{(p+q)\left(p^{2}+p q+q^{2}\right)}+B \leq & \frac{q}{p^{2}}(b-a) f\left({ }_{a} b_{p q}\right)+\frac{1}{p}(b-a) f(a) \\
& -\left(\frac{p+q}{p^{3}}\right) \int_{a}^{a} b_{p} f\left({ }_{a} x_{q^{2}}\right){ }_{a} d_{p, q} x \\
\leq & \frac{m p^{2} q^{2}(b-a)^{3}}{(p+q)\left(p^{2}+p q+q^{2}\right)}+A,
\end{aligned}
$$


where

$$
\begin{aligned}
A= & {\left[(b-a)\left({ }_{a} D_{p, q} f\left({ }_{a} b_{p}\right)-\frac{m p^{2}(b-a)}{p+q}\right)-\frac{1}{p}\left(f\left({ }_{a} b_{p q}\right)-f(a)\right)\right] } \\
& \times \frac{\left[\frac{1}{p}\left(f\left({ }_{a} b_{p q}\right)-f(a)\right)-(b-a)\left({ }_{a} D_{p, q} f(a)+\frac{m p q(b-a)}{p+q}\right)\right]}{{ }_{a} D_{p, q} f\left({ }_{a} b_{p}\right)-{ }_{a} D_{p, q} f(a)-m p(b-a)},
\end{aligned}
$$

and

$$
\begin{aligned}
B= & {\left[(b-a)\left({ }_{a} D_{p, q} f\left(a b_{p}\right)-\frac{M p^{2}(b-a)}{p+q}\right)-\frac{1}{p}\left(f\left({ }_{a} b_{p q}\right)-f(a)\right)\right] } \\
& \times \frac{\left[(b-a)\left({ }_{a} D_{p, q} f(a)+\frac{M p q(b-a)}{p+q}\right)-\frac{1}{p}\left(f\left({ }_{a} b_{p q}\right)-f(a)\right)\right]}{M p(b-a)-\left({ }_{a} D_{p, q} f\left({ }_{a} b_{p}\right)-{ }_{a} D_{p, q} f(a)\right)} .
\end{aligned}
$$

Proof. Observe that if $g$ and $h$ are continuous asynchronous functions on $J$ and $\phi$ is a non-negative continuous function, then Lemma 3 yields

$$
\begin{aligned}
& I_{p, q}^{a} \phi\left({ }_{a} b_{p}\right) I_{p, q}^{a}(\phi g h)\left({ }_{a} b_{p}\right)+I_{p, q}^{a} \phi\left({ }_{a} b_{p}\right) I_{p, q}^{a}(\phi g h)\left({ }_{a} b_{p}\right) \\
& \quad \leq I_{p, q}^{a}(\phi g)\left({ }_{a} b_{p}\right) I_{p, q}^{a}(\phi h)\left({ }_{a} b_{p}\right)+I_{p, q}^{a}(\phi g)\left({ }_{a} b_{p}\right) I_{p, q}^{a}(\phi h)\left({ }_{a} b_{p}\right) .
\end{aligned}
$$

That is,

$$
I_{p, q}^{a} \phi\left({ }_{a} b_{p}\right) I_{p, q}^{a}(\phi g h)\left({ }_{a} b_{p}\right) \leq I_{p, q}^{a}(\phi g)\left({ }_{a} b_{p}\right) I_{p, q}^{a}(\phi h)\left({ }_{a} b_{p}\right) .
$$

By Lemma 2, it implies that

$$
\begin{aligned}
\Omega & :=\int_{a}^{a} b_{p}(x-a)(b-x)_{a} D_{p, q}^{2} f(x){ }_{a} d_{p, q} x \\
& =\frac{q}{p^{2}}(b-a) f\left({ }_{a} b_{p q}\right)+\frac{1}{p}(b-a) f(a)-\left(\frac{p+q}{p^{3}}\right) \int_{a}^{a} b_{p} f\left({ }_{a} x_{q^{2}}\right){ }_{a} d_{p, q} x .
\end{aligned}
$$

Since ${ }_{a} D_{p, q}^{2} f$ is non-constant and $m \leq{ }_{a} D_{p, q}^{2} f \leq M$, we obtain

$$
\int_{a}^{a} b_{p}\left({ }_{a} D_{p, q}^{2} f(x)-m\right){ }_{a} d_{p, q} x>0 \text { and } \int_{a}^{a} b_{p}\left(M-{ }_{a} D_{p, q}^{2} f(x)\right){ }_{a} d_{p, q} x>0 .
$$

Consider

$$
\begin{aligned}
\int_{a}^{a b_{p}}(x-a)(b-x)\left({ }_{a} D_{p, q}^{2} f(x)-m\right){ }_{a} d_{p, q} x & =\Omega-m \int_{a}^{a b_{p}}(x-a)(b-x){ }_{a} d_{p, q} x \\
& =\Omega-\frac{m p^{2} q^{2}(b-a)^{3}}{(p+q)\left(p^{2}+p q+q^{2}\right)}
\end{aligned}
$$

and

$$
\begin{aligned}
& \int_{a}^{a b_{p}}(x-a)(b-x)\left(M-{ }_{a} D_{p, q}^{2} f(x)\right){ }_{a} d_{p, q} x=M \int_{a}^{a b_{p}}(x-a)(b-x){ }_{a} d_{p, q} x-\Omega \\
& =\frac{M p^{2} q^{2}(b-a)^{3}}{(p+q)\left(p^{2}+p q+q^{2}\right)}-\Omega .
\end{aligned}
$$

Observe that $x-a$ and $b-x$ are continuous asynchronous functions on $J$.

Substituting $g, h, \phi$ in (28) by $x-a, b-x$, and ${ }_{a} D_{p, q}^{2} f(x)-m$, respectively, we obtain 


$$
\begin{aligned}
& \int_{a}^{a b_{p}}(x-a)(b-x)\left({ }_{a} D_{p, q}^{2} f(x)-m\right){ }_{a} d_{p, q} x \\
& \leq \frac{\left(\int_{a}^{a b_{p}}(x-a)\left({ }_{a} D_{p, q}^{2} f(x)-m\right){ }_{a} d_{p, q} x\right)\left(\int_{a}^{a} b_{p}(b-x)\left({ }_{a} D_{p, q}^{2} f(x)-m\right){ }_{a} d_{p, q} x\right)}{\int_{a}^{a} b_{p}\left({ }_{a} D_{p, q}^{2} f(x)-m\right){ }_{a} d_{p, q} x} .
\end{aligned}
$$

Then, a direct calculation shows us that

$$
\int_{a}^{a} b_{p}\left({ }_{a} D_{p, q}^{2} f(x)-m\right){ }_{a} d_{p, q} x=\left({ }_{a} D_{p, q} f\left({ }_{a} b_{p}\right)-{ }_{a} D_{p, q} f(a)\right)-m p(b-a) .
$$

\section{Consider}

$$
\begin{aligned}
& \int_{a}^{a b_{p}}(x-a)\left({ }_{a} D_{p, q}^{2} f(x)-m\right){ }_{a} d_{p, q} x \\
& =\int_{a}^{a} b_{p}(x-a)_{a} D_{p, q}\left({ }_{a} D_{p, q} f(x)-m x\right){ }_{a} d_{p, q} x \\
& =\left[\frac{x-a}{p}\left({ }_{a} D_{p, q} f(x)-m x\right)\right]_{a}^{a b_{p}} \\
& -\int_{a}^{a} b_{p}\left({ }_{a} D_{p, q} f\left({ }_{a} x_{q}\right)-m\left({ }_{a} x_{q}\right)\right)\left({ }_{a} D_{p, q}\left(\frac{x-a}{p}\right)\right){ }_{a} d_{p, q} x \\
& =\left(\frac{a b_{p}-a}{p}\right)\left({ }_{a} D_{p, q} f\left({ }_{a} b_{p}\right)-m\left({ }_{a} b_{p}\right)\right) \\
& -\frac{1}{p} \int_{a}^{a} b_{p}\left({ }_{a} D_{p, q} f\left({ }_{a} x_{q}\right)-m\left({ }_{a} x_{q}\right)\right){ }_{a} d_{p, q} x \\
& =(b-a)\left({ }_{a} D_{p, q} f\left({ }_{a} b_{p}\right)-\frac{m p^{2}(b-a)}{p+q}\right)-\frac{1}{p}\left(f\left(a_{a} b_{p q}\right)-f(a)\right),
\end{aligned}
$$

and

$$
\begin{aligned}
& \int_{a}^{a b_{p}}(b-x)\left({ }_{a} D_{p, q}^{2} f(x)-m\right){ }_{a} d_{p, q} x \\
&=\int_{a}^{a} b_{p}(b-x){ }_{a} D_{p, q}\left({ }_{a} D_{p, q} f(x)-m x\right){ }_{a} d_{p, q} x \\
&= {\left[\frac{a b_{p}-x}{p}\left({ }_{a} D_{p, q} f(x)-m x\right)\right]_{a}{ }_{a} b_{p} } \\
&\left.-\int_{a}^{a}{ }_{a}{ }_{a}{ }_{a} D_{p, q} f\left({ }_{a} x_{q}\right)-m\left({ }_{a} x_{q}\right)\right)\left({ }_{a} D_{p, q}\left(\frac{{ }_{a} b_{p}-x}{p}\right)\right){ }_{a} d_{p, q} x \\
&=-\left(\frac{a_{p} b_{p}-a}{p}\right)\left({ }_{a} D_{p, q} f(a)-m a\right) \\
&+\frac{1}{p} \int_{a}^{a} b_{p}\left({ }_{a} D_{p, q} f\left({ }_{a} x_{q}\right)-m\left({ }_{a} x_{q}\right)\right){ }_{a} d_{p, q} x \\
&= \frac{1}{p}\left(f\left({ }_{a} b_{p q}\right)-f(a)\right)-(b-a)\left({ }_{a} D_{p, q} f(a)+\frac{m p q(b-a)}{p+q}\right) .
\end{aligned}
$$


By substituting (31)-(34) into (29), we get

$$
\begin{aligned}
\Omega- & \frac{m p^{2} q^{2}(b-a)^{3}}{(p+q)\left(p^{2}+p q+q^{2}\right)} \\
\leq & {\left[(b-a)\left({ }_{a} D_{p, q} f\left(a b_{p}\right)-\frac{m p^{2}(b-a)}{p+q}\right)-\frac{1}{p}\left(f\left({ }_{a} b_{p q}\right)-f(a)\right)\right] } \\
& \times \frac{\left[\frac{1}{p}\left(f\left({ }_{a} b_{p q}\right)-f(a)\right)-(b-a)\left({ }_{a} D_{p, q} f(a)+\frac{m p q(b-a)}{p+q}\right)\right]}{{ }_{a} D_{p, q} f\left({ }_{a} b_{p}\right)-{ }_{a} D_{p, q} f(a)-m p(b-a)} .
\end{aligned}
$$

Replacing $g, h, \phi$ in (28) by $x-a, b-x$, and $M-{ }_{a} D_{p, q}^{2} f(x)$, respectively, we obtain

$$
\begin{aligned}
& \int_{a}^{a b_{p}}(x-a)(b-x)\left(M-{ }_{a} D_{p, q}^{2} f(x)\right){ }_{a} d_{p, q} x
\end{aligned}
$$

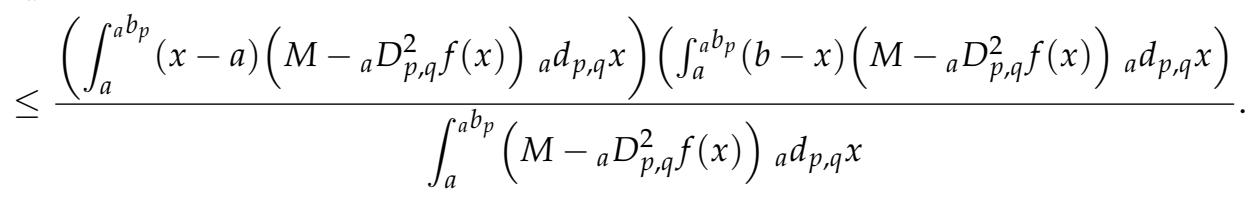

Then, a direct calculation shows us that

$$
\int_{a}^{a b_{p}}\left(M-{ }_{a} D_{p, q}^{2} f(x)\right){ }_{a} d_{p, q} x=M p(b-a)-\left({ }_{a} D_{p, q} f\left(a_{a} b_{p}\right)-{ }_{a} D_{p, q} f(a)\right) .
$$

\section{Consider}

$$
\begin{aligned}
\int_{a}^{a} b_{p} & x-a)\left(M-{ }_{a} D_{p, q}^{2} f(x)\right){ }_{a} d_{p, q} x \\
= & \int_{a}^{a} b_{p}(x-a)_{a} D_{p, q}\left(M x-{ }_{a} D_{p, q} f(x)\right){ }_{a} d_{p, q} x \\
= & {\left[\frac{x-a}{p}\left(M x-{ }_{a} D_{p, q} f(x)\right)\right]_{a}^{a} b_{p} } \\
& -\int_{a}^{a} b_{p}\left(M\left({ }_{a} x_{q}\right)-{ }_{a} D_{p, q} f\left({ }_{a} x_{q}\right)\right)\left({ }_{a} D_{p, q}\left(\frac{x-a}{p}\right)\right){ }_{a} d_{p, q} x \\
= & \left(\frac{{ }_{a} b_{p}-a}{p}\right)\left(M\left({ }_{a} b_{p}\right)-{ }_{a} D_{p, q} f\left({ }_{a} b_{p}\right)\right)-\frac{1}{p} \int_{a}^{a} b_{p}\left(M\left({ }_{a} x_{q}\right)-{ }_{a} D_{p, q} f\left({ }_{a} x_{q}\right)\right){ }_{a} d_{p, q} x \\
= & (b-a)\left(\frac{M p^{2}(b-a)}{p+q}-{ }_{a} D_{p, q} f\left({ }_{a} b_{p}\right)\right)+\frac{1}{p}\left(f\left({ }_{a} b_{p q}\right)-f(a)\right),
\end{aligned}
$$

and 


$$
\begin{aligned}
& \int_{a}^{a b_{p}}(b-x)\left(M-{ }_{a} D_{p, q}^{2} f(x)\right){ }_{a} d_{p, q} x \\
& =\int_{a}^{a} b_{p}(b-x)_{a} D_{p, q}\left(M x-{ }_{a} D_{p, q} f(x)\right){ }_{a} d_{p, q} x \\
& =\left[\frac{a b_{p}-x}{p}\left(M x-{ }_{a} D_{p, q} f(x)\right)\right]_{a}^{a b_{p}} \\
& -\int_{a}^{a b_{p}}\left(M\left({ }_{a} x_{q}\right)-{ }_{a} D_{p, q} f\left({ }_{a} x_{q}\right)\right)\left({ }_{a} D_{p, q}\left(\frac{a b_{p}-x}{p}\right)\right){ }_{a} d_{p, q} x
\end{aligned}
$$

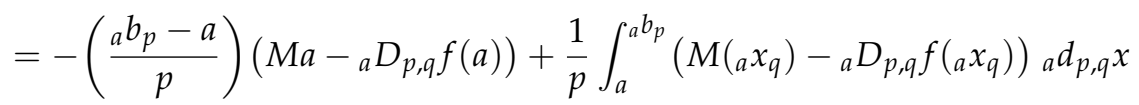

$$
\begin{aligned}
& =(b-a)\left({ }_{a} D_{p, q} f(a)+\frac{M p q(b-a)}{p+q}\right)-\frac{1}{p}\left(f\left(a b_{p q}\right)-f(a)\right) .
\end{aligned}
$$

By substituting (36)-(39) into (30), we get

$$
\begin{aligned}
& \frac{M p^{2} q^{2}(b-a)^{3}}{(p+q)\left(p^{2}+p q+q^{2}\right)}-\Omega \\
& \leq\left[\frac{1}{p}\left(f\left({ }_{a} b_{p q}\right)-f(a)\right)-(b-a)\left({ }_{a} D_{p, q} f\left({ }_{a} b_{p}\right)-\frac{M p^{2}(b-a)}{p+q}\right)\right] \\
& \quad \times \frac{\left[(b-a)\left({ }_{a} D_{p, q} f(a)+\frac{M p q(b-a)}{p+q}\right)-\frac{1}{p}\left(f\left({ }_{a} b_{p q}\right)-f(a)\right)\right]}{M p(b-a)-\left({ }_{a} D_{p, q} f\left(a b_{p}\right)-{ }_{a} D_{p, q} f(a)\right)} .
\end{aligned}
$$
proof.

By combining (35) and (40), we obtain the required inequality which accomplishes the

Corollary 1. Let $f: J \rightarrow \mathbb{R}$ be a twice q-differentiable with ${ }_{a} D_{q}^{2} f$ integrable on J. Assume that ${ }_{a} D_{q}^{2} f$ is non-constant with $m \leq{ }_{a} D_{q}^{2} f \leq M$. Then

$$
\begin{aligned}
\frac{M q^{2}(b-a)^{3}}{(1+q)\left(1+q+q^{2}\right)}+B & \leq q(b-a) f\left(a_{a} b_{q}\right)+(b-a) f(a)-(1+q) \int_{a}^{b} f\left({ }_{a} x_{q^{2}}\right){ }_{a} d_{q} x \\
& \leq \frac{m q^{2}(b-a)^{3}}{(1+q)\left(1+q+q^{2}\right)}+A
\end{aligned}
$$

where

$$
\begin{aligned}
A= & {\left[(b-a)\left({ }_{a} D_{q} f(b)-\frac{m(b-a)}{1+q}\right)-\left(f\left({ }_{a} b_{q}\right)-f(a)\right)\right] } \\
& \times \frac{\left[\left(f\left(a_{a} b_{q}\right)-f(a)\right)-(b-a)\left({ }_{a} D_{q} f(a)+\frac{m q(b-a)}{1+q}\right)\right]}{{ }_{a} D_{q} f(b)-{ }_{a} D_{q} f(a)-m(b-a)},
\end{aligned}
$$

and

$$
\begin{aligned}
B= & {\left[(b-a)\left({ }_{a} D_{q} f(b)-\frac{M(b-a)}{1+q}\right)-\left(f\left(a_{a} b_{q}\right)-f(a)\right)\right] } \\
& \times \frac{\left[(b-a)\left({ }_{a} D_{q} f(a)+\frac{M q(b-a)}{1+q}\right)-\left(f\left({ }_{a} b_{q}\right)-f(a)\right)\right]}{M(b-a)-\left({ }_{a} D_{q} f(b)-{ }_{a} D_{q} f(a)\right)} .
\end{aligned}
$$




\section{Conclusions}

We established some inequalities of Chebyshev-type inequalities by using $(p, q)$ integral, such as Chebyshev inequalities and the Fejér-like inequalities. Our work improves the results of Chebyshev-type quantum integral inequalities. By taking $q \rightarrow 1$ and $p=$ 1 , our results give classical inequalities. The $(p, q)$-integral inequalities deduced in the present research are very general and helpful in error estimations involved in various approximation processes. With these contributions, we hope these techniques and ideas established in this article will inspire the readers to explore the field of $(p, q)$-integral inequalities.

Author Contributions: Conceptualization, P.A. and S.K.N.; investigation, N.A., K.M.N. and K.N.; methodology, K.N.; validation, N.A., K.M.N., K.N., P.A. and S.K.N.; visualization, K.M.N., K.N., P.A. and S.K.N.; writing — original draft, N.A. and K.N.; writing—review and editing, K.N. All authors have read and agreed to the published version of the manuscript.

Funding: This research received funding support from the National Science, Research, and Innovation Fund (NSRF), Thailand.

Institutional Review Board Statement: Not applicable.

Informed Consent Statement: Not applicable.

Data Availability Statement: Not applicable.

Acknowledgments: We would like to thank the referees for their valuable comments and helpful advice on our manuscript.

Conflicts of Interest: The authors declare no conflict of interest.

\section{References}

1. Chebyshev, P.L. Sur les expressions approximati ves des integrales definies par les autres prises entre lesmemes limites. Proc. Math. Soc. Kharkov. 1882, 2, 93-98.

2. Kuang, J.C. Applied Inequalities; Shandong Sciences and Technologie Press: Shandong, China, 2004.

3. Mitrinović, D.S. Analytic Inequalities; Springer: Berlin, Germany, 1970.

4. Purohit, S.; Raina, R. Chebyshev-type inequalities for the Saigo fractional integrals and their $q$-analogues. J. Math. Inequal. 2013, 7, 239-249. [CrossRef]

5. Dahmani, Z. New inequalities in fractional integrals. Int. J. Nonlinear Sci. 2010, 9, 493-497.

6. Yang, W. Some new fractional quantum integral inequalities. Appl. Math. Lett. 2012, 25, 963-969. [CrossRef]

7. Choi, J.; Agarwal, P. Some new Saigo type fractional integral inequalities and their $q$-analogues. Abstr. Appl. Anal. 2014, $2014,11$. [CrossRef]

8. Baleanu, D.; Agarwal, P. Certain inequalities involving the fractional q-integral operators. Abstr. Appl. Anal. 2014, 2014, 10.

9. Brahim, K.; Taf, S. Some fractional integral inequalities in quantum calculus. J. Fract. Calc. Appl. 2013, 4, 245-250.

10. Chinchane, V.; Pachpatte, D. On some integral inequalities using Hadamard fractional integral. Malaya J. Mat. 2012, 1, $62-66$.

11. Jackson, F.H. On a q-definite integrals. Q. J. Pure Appl. Math. 1910, 41, 193-203.

12. Jackson, F.H. q-difference equations. Am. J. Math. 1910, 32, 305-314. [CrossRef]

13. Aslam, M.; Awan, M.U.; Noor, K.I. Quantum Ostrowski inequalities for $q$-differentiable convex function. J. Math. Inequal. 2016, 10, 1013-1018.

14. Aral, A.; Gupta, V.; Agarwal, R.P. Applications of q-Calculus in Operator Theory; Springer Science + Business Media: New York, NY, USA, 2013.

15. Gauchman, H. Integral inequalities in q-calculus. J. Comput. Appl. Math. 2002, 47, 281-300. [CrossRef]

16. Ahmad, B. Boundary-value problems for nonlinear third-order $q$-difference equations. Electron. J. Differ. Equ. 2011, 94, 1-7. [CrossRef]

17. Ahmad, B.; Alsaedi, A.; Ntouyas, S.K. A study of second-order $q$-difference equations with boundary conditions. Adv. Differ. Equ. 2012, 2012, 35. [CrossRef]

18. Ahmad, B.; Ntouyas, S.K.; Purnaras, I.K. Existence results for nonlinear $q$-difference equations with nonlocal boundary conditions. Comm. Appl. Nonlinear Anal. 2012, 19, 59-72.

19. Ahmad, B.; Nieto, J.J. On nonlocal boundary value problems of nonlinear $q$-difference equation. Adv. Differ. Equ. 2012, $2012,81$. [CrossRef]

20. Bukweli-Kyemba, J.D.; Hounkonnou, M.N. Quantum deformed algebra: Coherent states and special functions. arXiv 2013, arXiv:1301.0116v1. 
21. Bermudo, S.; Kórus, P.; Valdés, J.E.N. On $q$-Hermite-Hadamard inequalities for general convex functions. Acta Math. Hung. 2020, 162, 364-374. [CrossRef]

22. Chen, F.; Yang, W. Some new Chebyshev-type quantum integral inequalities on finite intervals. J. Comput. Anal. Appl. 2016, 21, 417-426.

23. Kac,V.; Cheung, P. Quantum Calculus; Springer: New York, NY, USA, 2002.

24. Tariboon, J.; Ntouyas, S.K. Quantum calculus on finite intervals and applications to impulsive difference equations. Adv. Differ. Equ. 2013, 2013, 282. [CrossRef]

25. Tariboon, J.; Ntouyas, S.K. Quantum integral inequalities on finite intervals. J. Inequal Appl. 2014, 2014, 121. [CrossRef]

26. Tunç, M.; Göv, E. Some integral inequalities via $(p, q)$-calculus on finite intervals. RGMIA Res. Rep. Coll. 2016, 19, 1-12. [CrossRef]

27. Tunç, M.; Göv, E. ( $p, q)$-integral inequalities. RGMIA Res. Rep. Coll. 2016, 19, 1-13.

28. Chakrabarti, R; Jagannathan, R. A $(p, q)$-oscillator realization of two-parameter quantum algebras. J. Phys. A Math. Gen. 1991, 24, L711-L718. [CrossRef]

29. Prabseang, J.; Nonlaopon, K.; Tariboon, J. $(p, q)$-Hermite-Hadamard inequalities for double integral and $(p, q)$-differentiable convex functions. Axioms 2019, 8, 68. [CrossRef]

30. Kalsoom, H.; Amer, M.; Junjua, M.D.; Hassain, S.; Shahxadi, G. $(p, q)$-estimates of Hermite-Hadamard-type inequalities for coordinated convex and quasi convex function. Mathematics 2019, 7, 683. [CrossRef]

31. Hounkonnou, M.N.; Désiré, J.; Kyemba, B. R. ( $p, q)$-calculus: Differentiation and integration. SUT J. Math. 2013, $49,145-167$.

32. Sadjang, P.N. On the fundamental theorem of $(p, q)$-calculus and some $(p, q)$-Taylor formulas. Results Math. 2018, 73, 39. [CrossRef]

33. Chu, Y.M.; Awan, M.U.; Talib, S.; Noor, M.A.; Noor, K.I. New post-quantum analogues of Ostrowski-type inequalities using new definitions of left-right $(p, q)$-derivatives and definite integrals. Adv. Differ. Equ. 2020, 2020, 634. [CrossRef]

34. Kalsoom, H.; Rashid, S.; Tdrees, M.; Safdar, F.; Akram, S.; Baleanu, D.; Chu, Y.M. Post quantum inequalities of Hermite-Hadamardtype associated with co-ordinated higher-order generalized strongly pre-index and quasi-pre-index mappings. Symmetry 2020, 12, 443. [CrossRef]

35. Kunt, M.; Iscan, I.; Alp, N.; Sarikaya, M.Z. $(p, q)$-Hermite-Hadamard and $(p, q)$-estimates for midpoint type inequalities via convex and quasi-convex functions. RACSAM 2018, 112, 969-992. [CrossRef]

36. Ali, M.A.; Budak, H.; Kalsoom, H.; Chu, Y.M. Post-quantum Hermite-Hadamard inequalities involving newly defined $(p, q)$ integral. Authorea 2020. [CrossRef]

37. Thongjob, S.; Nonlaopon, K.; Ntouyas, S.K. Some $(p, q)$-Hardy type inequalities for $(p, q)$-integrable functions. AIMS Math. 2020, 6, 77-89. [CrossRef]

38. Wannalookkhee, F.S.; Nonlaopon, K.; Tariboon, J.; Ntouyas, S.K. On Hermite-Hadamard type inequalities for coordinated convex functions via $(p, q)$-calculus. Mathematics 2021, 9, 698. [CrossRef]

39. Arunrat, N.; Nakprasit, K. M.; Nonlaopon, K.; Tariboon, J.; Ntouyas, S. K. On Fejér type inequalities via $(p, q)$-calculus. Symmetry 2021, 13, 953. [CrossRef] 